\title{
Preoperative Diagnosis of Adult Intussusception Caused by Small Bowel Lipoma
}

\author{
Hiroaki Shiba ${ }^{a}$ Yoshinobu Mitsuyama ${ }^{a}$ Ken Hanyu ${ }^{a}$ \\ Kenji Ikeuchi $^{\mathrm{b}}$ Hirotaka Hayashic Katsuhiko Yanaga $^{\mathrm{a}}$

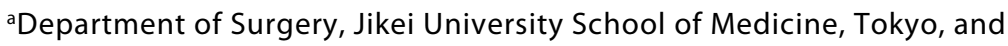 \\ Departments of 'burgery and 'Pathology, Nishisaitama-Chuo National Hospital, \\ National Hospital Organization, Saitama, Japan
}

\section{Key Words}

Adult · Intussusception · Lipoma

\begin{abstract}
Adult intussusception is rare, accounting for only $5 \%$ of all intussusceptions, for which preoperative diagnosis is difficult. We herein report a preoperatively diagnosed case of adult intussusception caused by a small bowel lipoma. A 33-year-old man was admitted to our hospital with three weeks history of colicky epigastric pain. Computed tomography revealed thickening of the ileal wall suggestive of intussusception. Colonoscopy revealed an ileocolic intussusception. Barium enema for reduction of ileocolic intussusception demonstrated a small bowel tumor in the ileum $15 \mathrm{~cm}$ proximal to the ileocecal valve. The intussusception was reduced, and the patient underwent partial resection of the ileum encompassing the small bowel tumor. Histological findings confirmed the diagnosis of lipoma of the small bowel. The patient made a satisfactory recovery and remains well.
\end{abstract}

\section{Introduction}

Intussusception is not a rare disease in children, however in adults it accounts for only $1 \%$ of bowel obstructions [1], and 5\% of all intussusceptions [2]. Preoperative diagnosis of adult intussusception is difficult and often confirmed at laparotomy [3]. We herein report a preoperatively diagnosed case of adult intussusception caused by a small bowel lipoma.

\section{Case Report}

A 33-year-old man was admitted to our hospital with three weeks history of colicky epigastric pain. Before admission the patient had been hospitalized at another hospital, but the cause of the repeated 
colicky epigastric pain had not been identified. The patient had no significant past medical history of illnesses nor any hospitalization otherwise. On physical examination, the abdomen was flat and no tumor was palpable. Computed tomography revealed thickening of the ileal wall suggesting intussusception (fig. 1). Colonoscopy revealed ileocolic intussusception, but no obvious cause was identified (fig. 2). Barium enema for reduction of the ileocolic intussusception showed a small bowel tumor in the ileum $15 \mathrm{~cm}$ proximal to the ileocecal valve (fig. 3). Reduction of the intussusception under barium enema was succeesful, and the patient underwent laparotomy the next day. However, recurrent ileocolic intussusception was found, for which a manual reduction and a partial resection of the ileum encompassing the small bowel tumor in the ileum $15 \mathrm{~cm}$ proximal to the ileocecal valve was performed. The resected specimen showed a soft and yellowish-white submucosal tumor with a diameter of $40 \times 25 \mathrm{~mm}$. Histological findings revealed fat cells proliferating in the submucosal layer and confirmed the diagnosis of lipoma of the small bowel. The patient made a satisfactory recovery and remains well 2 years after surgery.

\section{Discussion}

Adult intussusception is rare and usually caused by a tumor acting as the apex of the intussusception. Therefore, such a condition should indicate the possibility of the presence of malignancy in the bowel. However, it has been reported that $52-80 \%$ of cases of adult small bowel intussusception are caused by benign entities $[4,5]$, such as lipoma, hamartomatous polyp, inflammatory polyp, hyperplastic polyp, Meckel's diverticulum or surgery-related lesions. Lipoma is the most common benign tumor in both small and large bowel intussusception [5]. Because benign tumors of the small bowel are commonly polypoid-shaped, the intussusception usually develops before the tumor has grown to a size sufficient to obstruct the passage of the small intestinal contents [6].

Small intestinal tumors are rare, accounting for $1-2 \%$ of all gastrointestinal tract tumors [7]. Moreover, preoperative diagnosis is often difficult because of the lack of specific clinical symptoms and difficulty with the examination of the small intestine. Computed tomography and ultrasonography of the abdomen are helpful for the diagnosis of intussusception and intestinal lipoma $[4,8]$. However, further examinations such as barium enema or endoscopic ultrasonography are useful in cases of difficult diagnosis [9].

It is accepted that gentle preoperative or operative reduction can be attempted safely to avoid unnecessary operation [10]. Laparoscopic surgery for intussusception is increasing, and successful reduction of intussusception recently has been reported [11]. Although more technically difficult than laparotomy, with an increased risk of perforation and dissemination and metastasis in cases of malignancies or with intestinal dilatation, laparoscopic surgery seems to become a procedure of choice for intussusception caused by benign intestinal tumors in the future. 
Fig. 1. Computed tomography revealed thickening of the ileal wall and suggested intussusception (arrow).

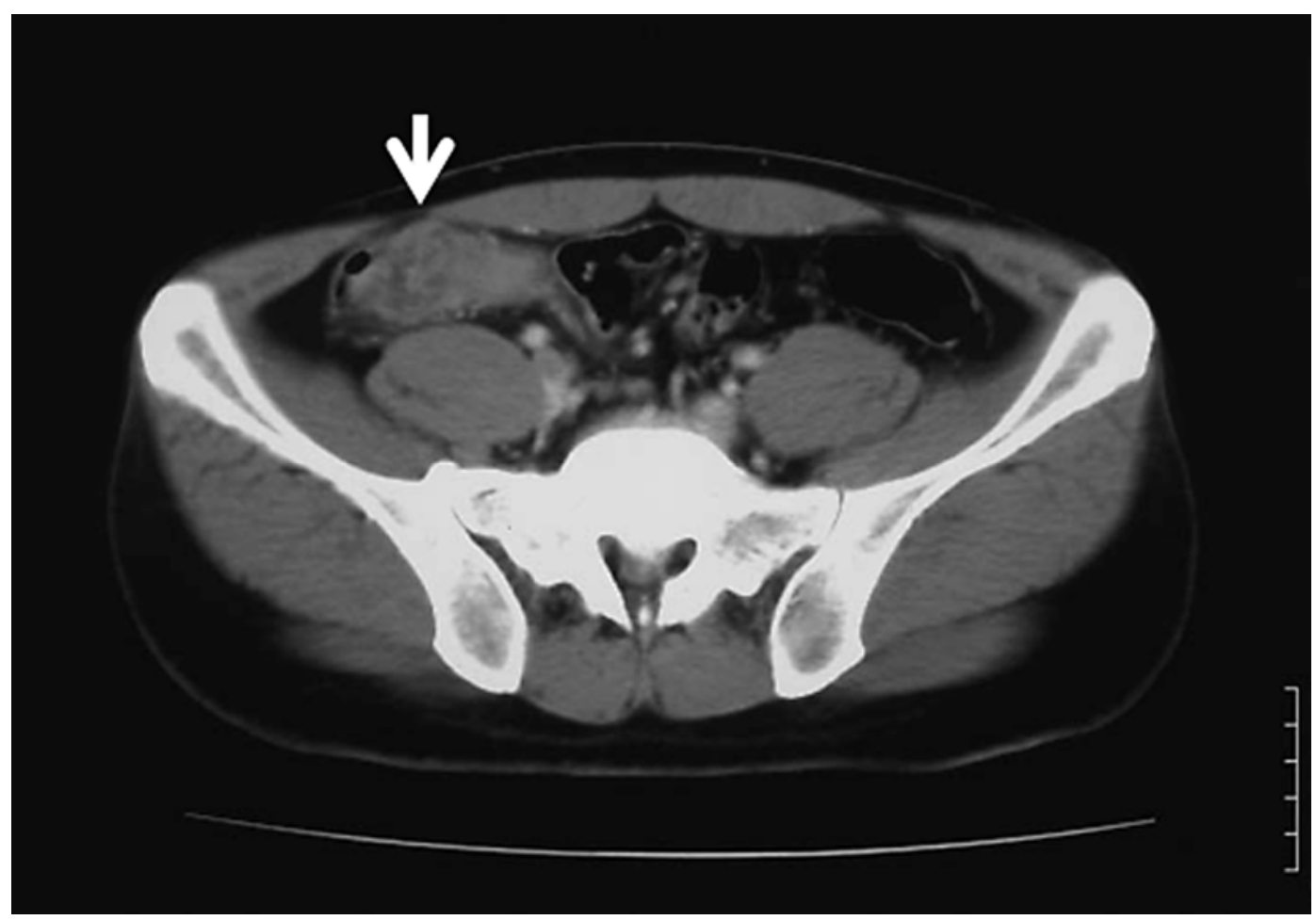

Fig. 2. Colonoscopy revealed an ileocolic intussusception, but no obvious cause of intussusception.

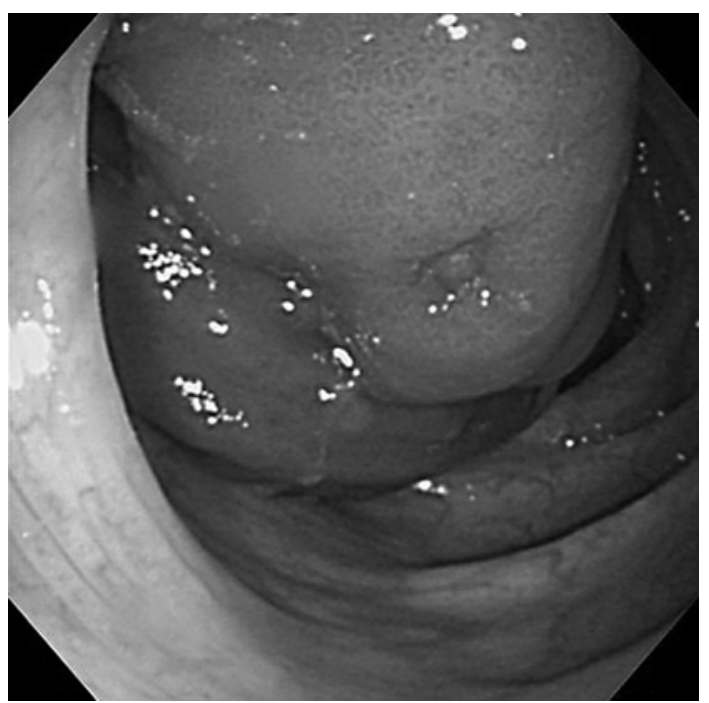


Fig. 3. Barium enema, for reduction of ileocolic intussusception, showed a small bowel tumor in the ileum $15 \mathrm{~cm}$ proximal to the ileocecal valve (arrow).

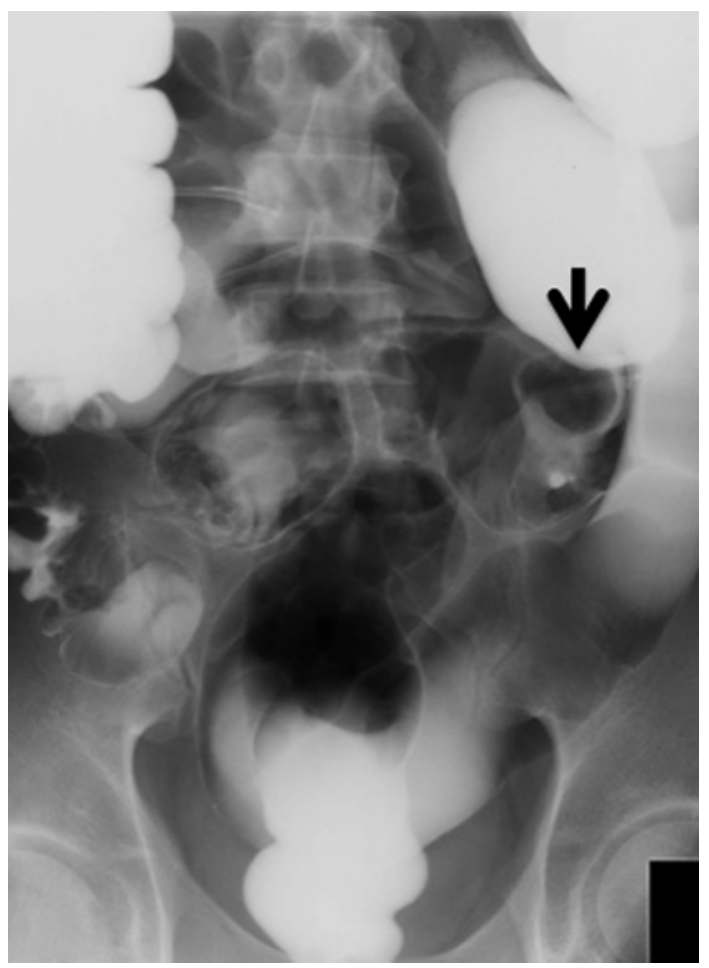




\section{References}

1 Stubenbord WT, Thornjarnarson B: Intussusception in adults. Ann Surg 1970;172:306-310.

2 Nagorney DM, Sarr MG, McIlrath DC: Surgical management of intussusception in the adult. Ann Surg 1981;193:230-236.

3 Meshikhes AW, Al-Momen SA, Al Talaq FT, Al-Jaroof AH: Adult intussusception caused by a lipoma in the small bowel: report of a case. Surg Today 2005;35:161-163.

4 Azar T, Berger DL: Adult intussusception. Ann Surg 1997;226:134-138.

5 Chiang JM, Lin YS: Tumor spectrum of adult intussusception. J Surg Oncol 2008;98:444-447.

6 Wood KF: Ileo-colic intussusception in an adult. Br Med J 1954;2:970.

7 Good CA: Tumor of the small intestine. AJR Am J Roentgenol 1963;89:695-705.

8 Urbano J, Serantes A, Hernandez L, Turegano F: Lipoma-induced jejunojejunal intussusception: US and CT diagnosis. Abdom Imaging 1996;21:522-524.

9 Watanabe F, Honda S, Kubota H, et al: Preoperative diagnosis of ileal lipoma by endoscopic ultrasonography probe. J Clin Gastroenterol 2000;31:245-247.

10 Khurrum Baig M, Hussain S, Wise M, Wexner SD: Controversies in the treatment of adult long ileo-caecal intussusception: case report. Am Surg 2000;66:742-743.

11 Tsushimi T, Matsui N, Kurazumi H, et al: Laparoscopic resection of an ileal lipoma: report of a case. Surg Today 2006;36:1007-1010. 